\title{
Modelo Semântico e Pedagógico de Tutoria para Gestão Pessoal da Carreira: Análise e Resultados Preliminares
}

\author{
Gustavo Henrique de Souza ${ }^{1}$, João Carlos Gluz ${ }^{1}$ \\ ${ }^{1}$ Pós-Graduação em Computação Aplicada (PPGCA) - Universidade do Vale do Rio dos \\ Sinos (UNISINOS) - Av. Unisinos 950 - 93022-000 - São Leopoldo - RS - Brazil \\ gtvosouza@hotmail.com, jcgluz@unisinos.br
}

\begin{abstract}
Career management issues have undergone significant changes in recent decades, bringing new perspectives of the prominence of the individual over the organization. The career shall be understood as a sequence consisting of all the professional experiences of the individual throughout his life. This article proposes an ontological model and a mentoring system for personal career management aimed at learning meta-cognitive career management strategies. The paper closes with the presentation of the preliminary results of the empirical evaluation and the usability of the system, which show good evidence of the usefulness of the system to help with professional life and critical thinking about how to manage one's career.
\end{abstract}

Resumo. $O$ assunto carreira passou por significativas evoluções nas últimas décadas, trazendo uma nova perspectiva da proeminência do indivíduo sobre a organização. A carreira passa a ser compreendida como uma sequência constituída por todas as experiências profissionais do indivíduo ao longo de sua vida. Este artigo propõe um modelo ontológico e um sistema de tutoria para gestão de carreiras voltado à aprendizagem de estratégias metacognitivas de gestão da carreira. O trabalho se encerra com a apresentação dos resultados preliminares da avaliação empírica e da usabilidade do sistema, que mostram boas evidências da utilidade do sistema para auxiliar na vida profissional e no pensamento crítico sobre como gerir a própria carreira.

\section{Introdução}

A perspectiva tradicional do desenvolvimento da carreira baseada na proeminência da organização sobre o indivíduo vem sendo discutida por várias pesquisas nas últimas décadas (Crites, 1976). Neste contexto, a literatura mais recente não se foca apenas na organização como o objeto principal da pesquisa (Rosa et al., 2015), mas torna cada vez mais saliente o papel do indivíduo, como um ator que tem a necessidade de saber como conduzir de forma eficaz sua própria carreira (King, 2001; King, 2004; Pinto 2010). No ambiente profissional e econômico atual, a capacidade de gerir sua própria carreira e de estar preparado para aprender novas competências e habilidades durante toda a vida profissional (King, 2001), se tornaram importantes diferenciais.

Isso leva o problema para um contexto educacional, dadas as necessidades de aprendizagem constantes de novas capacidades e competências, incluindo as habilidades de gestão da própria carreira. Metodologias derivadas de métodos educacionais, como mentoring (tutoria) ou coaching (orientação), vem sendo aplicadas com sucesso na tutoria para o desenvolvimento pessoal da carreira, através do relacionamento com um tutor ou orientador com uma ampla e sólida experiência profissional (Palacios, 2006; Raggins e Kram, 2007). Apesar de resultados positivos, essas técnicas exigem um custo muito alto pela forma de sua operacionalização: tutoria e orientação são técnicas personalizadas que requerem profissionais específicos com boa experiência acumulada (Oluwabunmi, 2016). Isso leva a análise de possibilidades de aplicação de ambientes 
VI Congresso Brasileiro de Informática na Educação (CBIE 2017)

Anais do XXVIII Simpósio Brasileiro de Informática na Educação (SBIE 2017)

inteligentes de ensino para ajudar nesse problema (Weerasinghe et al., 2016), através da criação de sistemas inteligentes para tutoria e orientação de carreiras (IMS - Intelligent Mentoring Systems).

Segundo (Du Boulay, 2016), a principal diferença entre os IMS e os tradicionais sistemas tutores inteligentes (ITS - Intelligent Tutoring Systems) é o período de tempo muito mais longo de uso do IMS por um profissional, quando comparado ao período de tempo que os ITS são utilizados por seus alunos. A tutoria e orientação de carreiras requerem um uso continuado mais longo que os processos de ensino usuais, onde os ITS são comumente aplicados (Du Boulay, 2016). Isso traz questões importantes, ainda não resolvidas para o projeto dos IMS. O uso do IMS por um tempo mais longo não é apenas consequência de um investimento maior em conteúdos e conhecimentos a serem estudados, mas também porque a (re)orientação de carreira, requer não apenas novos conhecimentos, mas possivelmente novas expectativas, objetivos e valores, com a consequente necessidade de análise crítica e possível revisão das expectativas, objetivos e valores atuais. Essa questão é importante porque leva o IMS a ensinar capacidades de meta-conhecimento e meta-cognição. $\mathrm{O}$ alunos (ou profissionais) têm que buscar respostas para as questões: o que realmente é preciso aprender para (re)orientar a carreira e quais objetivos e expectativas são importantes neste processo.

O presente trabalho busca oferecer respostas para essas questões, propondo um modelo semântico e pedagógico a ser usado na construção de um IMS voltado para uma área profissional específica. Este modelo aplica o estado da arte em representação lógica de conhecimentos, baseada em ontologias OWL, para definir formalmente o processo de gestão de carreiras de acordo com o modelo de gestão pessoal de carreiras proposto por King (2001) e com o modelo cognitivo dos conhecimentos, habilidades e atitudes de uma dada competência (Carbone, 2006). Em termos pedagógicos e dinâmicos, o modelo formal de carreiras e competências é combinado com um processo de ensino e aprendizagem organizado em situações-problemas (Perrenoud, 2000) voltadas a identificação, compreensão e reflexão das competências, habilidades e atitudes necessárias para a evolução da carreira individual, ou seja, voltado para a aprendizagem de estratégias de meta-cognição e meta-conhecimento.

Para fins de avaliação empírica do modelo semântico ontológico de carreiras e competências e do modelo pedagógico e dinâmico de situacões-problema. foi construído um protótipo de IMS. denominada de MaxMentor que incorpora cenários de mudanca de carreira na área de Tecnologia da Informacão (TI). Tais cenários foram testados empiricamente por profissionais com variados níveis de experiência na área profissional escolhida. para fins de avaliacão de usabilidade e de possíveis impactos na aprendizagem de gestão de carreiras. A questão de pesquisa. relacionada a possibilidade de aprendizagem de estratégias meta-cognitivas na gestão de carreiras, foi avaliada por um especialista em coaching de carreiras.

\section{Trabalhos relacionados}

Na pesquisa sobre o estado da arte. inicialmente foram investigados artigos relacionados aos modelos de gestão e representacão de competências. O artigo (Oliveira e Gluz. 2010) define um modelo computacional para competências humanas com o obietivo de aiudar gerentes a selecionar profissionais para a integracão em uma equipe de desenvolvimento de software. O principal resultado obtido é mostrar que um modelo competências baseado em Conhecimentos. Habilidades e Atitudes, pode ser construído através de agentes cognitivos, baseados na arquitetura BDI (do inglês Beliefs - Desires Intentions). O trabalho (Rosa et al. 2015) apresenta o sistema MultCComp que tira proveito de contextos presentes e contextos anteriores para ajudar trabalhadores na melhoria de suas competências. Tais contextos são usados para extrair informações que permitem a detecção automática dos avanços nas competências. 
Após foram analisados pesquisas sobre IMS realizadas no primeiro workshop internacional nessa área (IMS 2016). Além de serem bastante atuais, estes trabalhos oferecem uma amostra significativa de como técnicas de aprendizagem estão sendo usadas no contexto de tutoria e orientação profissional de carreiras. O trabalho (Oluwabunmi et al. 2016) quais os efeitos da confiança interpessoal na relação entre grupos de pares de orientação. Os resultados mostraram que a pontuação de confiança interpessoal é um preditor fraco de seu comportamento de classificação como mentor. $\mathrm{O}$ trabalho (Popescu et al., 2016) busca unir três métodos adaptativos de aprendizagem: agrupamento de estudantes, agrupamento de aprendizagem, incluindo CGL (do inglês Class room Group Learning) e OGL (do inglês Online-based Group Learning) e orientação em pares. A pesquisa de Dicheva (2016) apoia duas estratégias de aprendizagem, a primeira é denominada de sala de aula invertida (do inglês flipped class room) e a segunda estratégia é a gamificação. Esses trabalhos trazem uma serie de abordagens de se trabalhar com gestão de carreiras e competências. A Tabela 2 mostra essas abordagens, em comparação ao sistema MaxMentor proposto neste trabalho. A principal contribuição do MaxMentor, além de unir a gestão de carreiras e de competências através de um modelo ontológico, é a possibilidade deste sistema trabalhar com a aprendizagem de estratégias meta-cognitivas na gestão de carreiras. Em particular, exceto pelo artigo (Dimitrova, 2016), os demais artigos do workshop IMS 2016 não consideram os aspectos de meta-conhecimento e meta-cognição abordados no presente trabalho.

Tabela 2: Tabela comparativa de conceitos.

\begin{tabular}{|c|c|c|c|c|c|c|c|c|c|c|c|c|c|}
\hline & CHA & Gamif. & GPC & GCE & Contextos & BDI & $\begin{array}{c}\text { Meta- } \\
\text { Cognição }\end{array}$ & IMCS & $\begin{array}{c}\text { Aula } \\
\text { Invertida }\end{array}$ & $\begin{array}{c}\text { Efeitos de } \\
\text { Confiança }\end{array}$ & M.A.A & Grupos & Agentes \\
\hline MaxMentor & $\mathrm{X}$ & & $\mathrm{X}$ & & & $\mathrm{X}$ & $\mathrm{X}$ & $\mathrm{X}$ & & & & & $\mathrm{X}$ \\
\hline Rosa et al. (2015) & & & & $\mathrm{X}$ & $\mathrm{X}$ & & & & & & & & \\
\hline Oliveira e Gluz(2010) & $\mathrm{X}$ & & & $\mathrm{X}$ & & $\mathrm{X}$ & & & & & & & $\mathrm{X}$ \\
\hline Dicheva(2016) & & $\mathrm{X}$ & $\mathrm{X}$ & & & & & & $\mathrm{X}$ & & & & \\
\hline Oluwabunmi(2016) & & & $\mathrm{X}$ & & & & & $\mathrm{X}$ & & $\mathrm{X}$ & & $\mathrm{X}$ & \\
\hline Popescu et al. (2016) & & $\mathrm{X}$ & & & & & $\mathrm{X}$ & & & $\mathrm{X}$ & $\mathrm{X}$ & \\
\hline Dimitrova (2016) & & & $\mathrm{X}$ & & & & $\mathrm{X}$ & $\mathrm{X}$ & & & & & \\
\hline
\end{tabular}

\section{Modelo Ontológico de Gestão de Carreiras}

O modelo de gestão pessoal da carreira proposto por (King, 2001), se tornou uma alternativa eficaz aos modelos tradicionais de desenvolvimento e planejamento de carreira com o propósito de apoiar adultos trabalhadores, independentemente da situação profissional, na gestão pessoal de suas carreiras (Pinto, 2010). A estrutura teórica feita por King engloba a natureza (comportamentos) da gestão pessoal de carreiras, e também as variáveis antecedentes (causas) e consequentes (efeitos). Competência, no sentido adotado neste trabalho, trata-se da capacidade e da suficiência que alguém tem para resolver um assunto com base em sua aptidão (ver Dicionário Aurélio, (Ferreira, 2010). Durand (1998), detalha este conceito, definindo que competências seguem três dimensões: Conhecimentos, Habilidades e Atitudes (CHA), englobando não só conhecimentos técnicos, mas também cognição e atitudes relacionadas ao trabalho. Assim competência diz respeito ao conjunto de conhecimentos, habilidades e atitudes para a execução de uma dada tarefa.

Com base no modelo King e na análise de competências dos planos de carreira da área de TI, foi criado um modelo ontológico para o processo de Gestão Pessoal de Carreiras (GPC) nessa área. As classes CausasComportamento, ComportamentoGestao e ConsequenciasComportamento, dizem respeito do modelo King de gestão pessoal de carreira. A classe Competencias é a representação dos conceitos de competências relacionados ao modelo CHA. A classe Funcao representa as funções identificadas nos planos de carreira da área de TI. A classe Sujeito representa o indivíduo interessado em gerir sua carreira. A definição dos planos de carreira na ontologia GPC foi baseada na análise empírica dos planos de carreira de três empresas da área de TI, já que não existe 
VI Congresso Brasileiro de Informática na Educação (CBIE 2017)

Anais do XXVIII Simpósio Brasileiro de Informática na Educação (SBIE 2017)

um órgão oficial para regulamentar esta carreira. A Figura 1 apresenta os níveis superiores da hierarquia de classes do modelo GPC, incluindo a hierarquia de classes correspondentes as funções e níveis de experiência da área de TI.
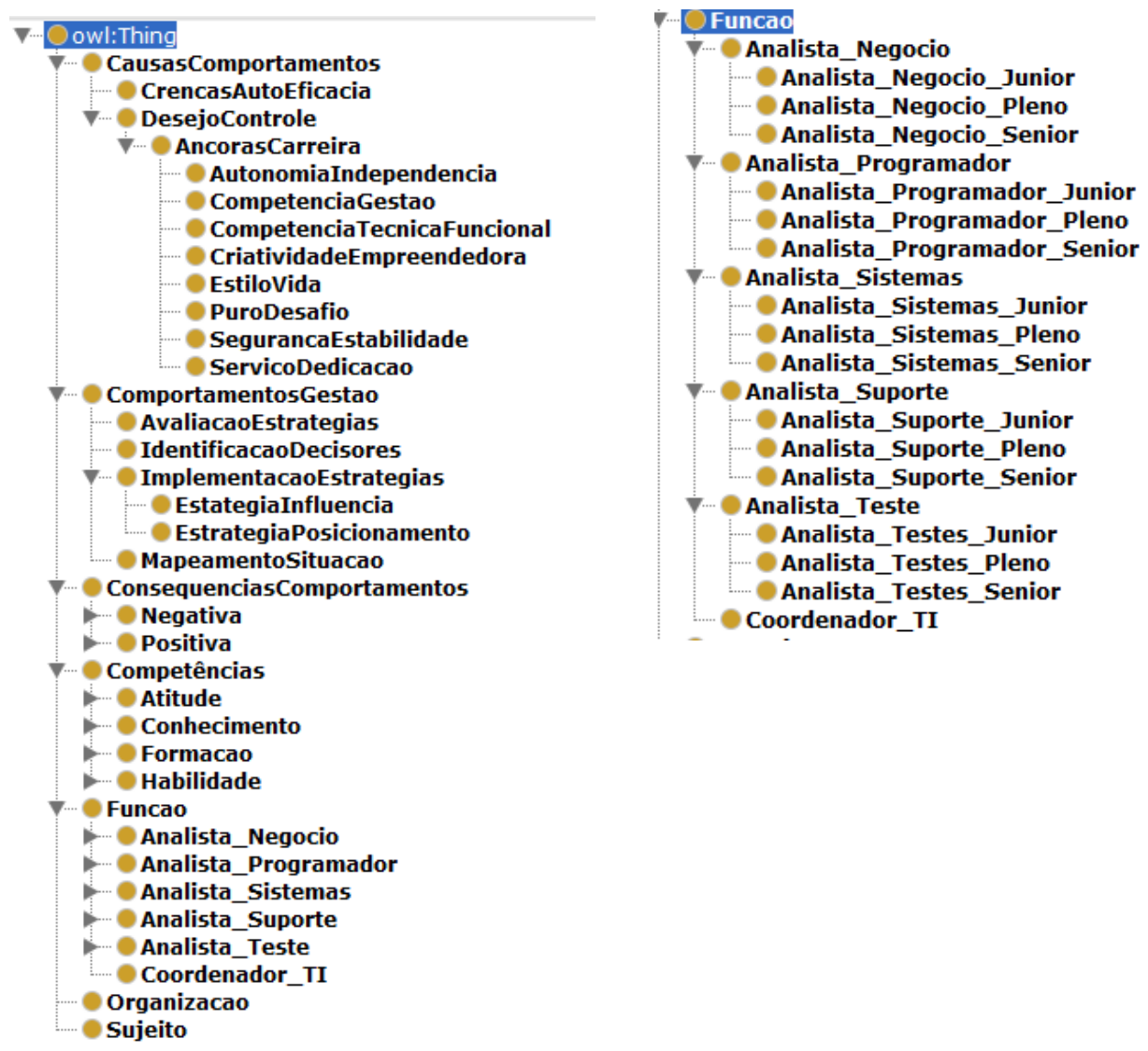

Figura 1: Níveis Gerais da Hierarquia de Classes da Ontologia GPC

Para a definição dos planos de carreira foram buscados dados de empresas situadas em cidades com perfis econômicos e demográficos distintos. Duas cidades tem economia diversificada e são de porte grande com mais de um milhão de habitantes A terceira cidade tem tamanho médio, com um perfil econômico especializado. Também se buscou obter dados de empresas de TI com diversos tamanhos, incluindo uma empresa pequena de 25 funcionários, uma empresa média de 300 funcionários e uma empresa de grande porte com aproximadamente 12.000 funcionários.

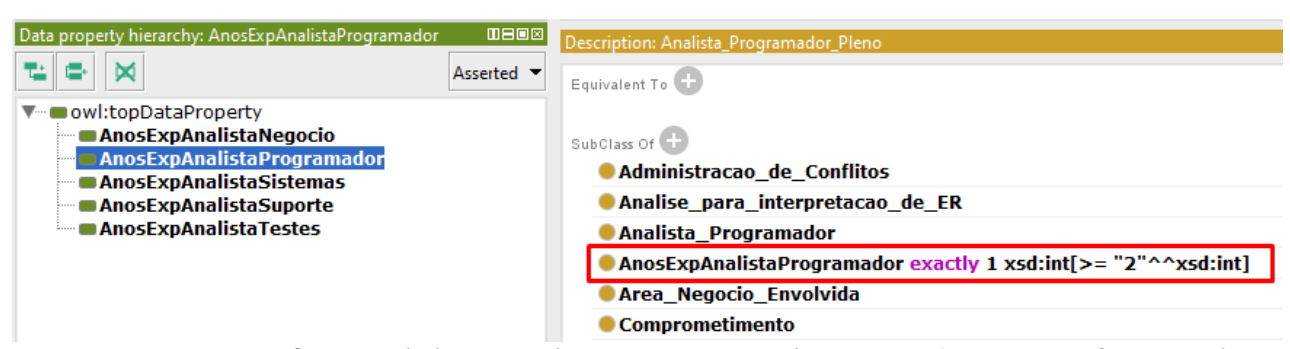

Figura 2: Modelagem dos requisitos de experiência profissional

Os dados obtidos dessas empresas mostraram seis funções que se destacam pela similaridade de competências exigidas: Analista de Negócio, Analista de Sistemas, Analista Programador, Analista de Testes, Analista de Suporte e Coordenador de TI. Exceto para a função de Coordenador de TI, todas as demais funções se dividem em três níveis de experiência: Sênior, Pleno e Junior. Para cada uma dessas funções foram mapeadas as competências definidas de acordo com o modelo $\mathrm{CHA}$, necessárias para a 
atuação de um profissional no exercício da função, bem como o tempo de exercício necessário para cada nível de experiência. Propriedades de dados OWL definem os requisitos específicas de cada nível e função. Por exemplo um Analista Programador Pleno, terá no mínimo dois anos de experiência como Analista de Programador. A Figura 2 mostra como esse requisito é modelado na ontologia GPC.

\section{Processo de Tutoria no MaxMentor}

O processo de tutoria do MaxMentor começa pela apresentação de um formulário para a obtenção dos dados a respeito da posição atual do profissional (aluno) em relação a sua carreira. Logo após, é necessário que o profissional sendo orientado indique quais são seus objetivos de carreira. A partir destes dados iniciais, o MaxMentor, irá sugerir possíveis objetivos de carreira de acordo com a possibilidade de atingimento das competências, através de um processo de inferência que primeiro confronta as competências existentes contra as competências necessárias de se atingir e então faz uma análise de meios e fins para identificar as opções com menores pendências a atingir e que oferecem os maiores ganhos ao profissional.

A Figura 3 mostra um exemplo hipotético de avaliação do MaxMentor. A opção em amarelo oferece o menor número de pendências, ou seja, neste exemplo o Sujeito A mudaria apenas seu nível de Júnior para Pleno. Ela está em amarelo porque, apesar dessa mudança ter apenas quatro pendências (novas competências a atingir), esta função está mais próxima de sua âncora de carreira e oferece menos ganhos salariais. A opção em verde, classificada como "Bom", apresenta um número mais elevado de pendências, porém, todas são possíveis de serem alcançadas, ou seja, levará mais tempo e esforço para alcançar todas as competências necessárias, mas o objetivo não apenas estará de acordo com sua ancora de carreira, mas permitirá um avanço salarial mais significativo. A opção em vermelho representa um salto muito grande na carreira do indivíduo, sendo classificada como "Ruim" por não ir de encontro com sua âncora de carreira e exigir um grande tempo de experiência em competências ainda não adquiridas.

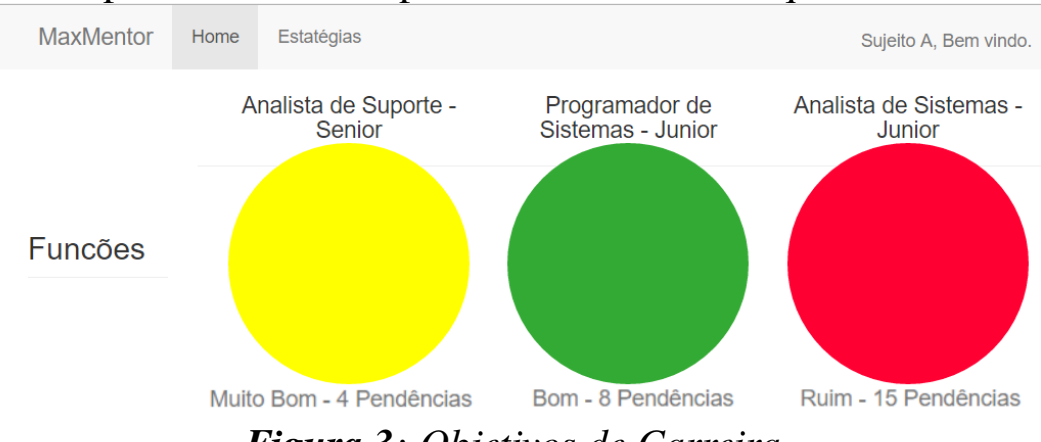

Figura 3: Objetivos de Carreira

Após a escolha do objetivo de carreira, começa o processo de orientação para o planejamento dos passos para atingir esse objetivo que segue a abordagem pedagógica de situações-problema proposta por Perrenoud (2000). Nesta abordagem, quando uma pessoa enfrenta a resolução de um desafio, ela é colocada diante de um obstáculo cognitivo, que poderá ser superado através de um processo de aprendizagem. Competência, de acordo com Perrenoud (2000), é a possibilidade que um indivíduo tem de mobilizar recursos (saber, saber fazer, e saber ser) para resolver uma situação que oferece um problema, ou seja, uma situação-problema. Para tanto, o MaxMentor apresenta um número variável de situações-problema de acordo com o objetivo do aluno, que são divididas em três partes de acordo com o modelo CHA de competências. Assim cada situação problema apresenta pendências ao aluno para adquirir conhecimentos e habilidades ou formar atitudes. Na medida em que o aluno seleciona uma situação problema, o MaxMentor exibe todas as competências necessárias para determinado objetivo, questionando se o aluno acredita ter ou não aquela competência. 
VI Congresso Brasileiro de Informática na Educação (CBIE 2017)

Anais do XXVIII Simpósio Brasileiro de Informática na Educação (SBIE 2017)

Esta etapa está ligada com às crenças de auto eficácia do indivíduo, sendo necessário fazer uma avaliação sobre si mesmo, para que no futuro, de alguma forma, possa implementar uma estratégia, sendo ela de influência ou de posicionamento, conforme descrito por King.

$\mathrm{Na}$ sequência da tutoria, conforme o aluno identifique que precisa agir diante a alguma competência, o MaxMentor possibilita duas formas para a implementação de estratégias, a primeira se refere as estratégias propostas pelo tutor e a segunda são estratégias personalizadas. A Figura 5 mostra um exemplo de estratégia personalizada.

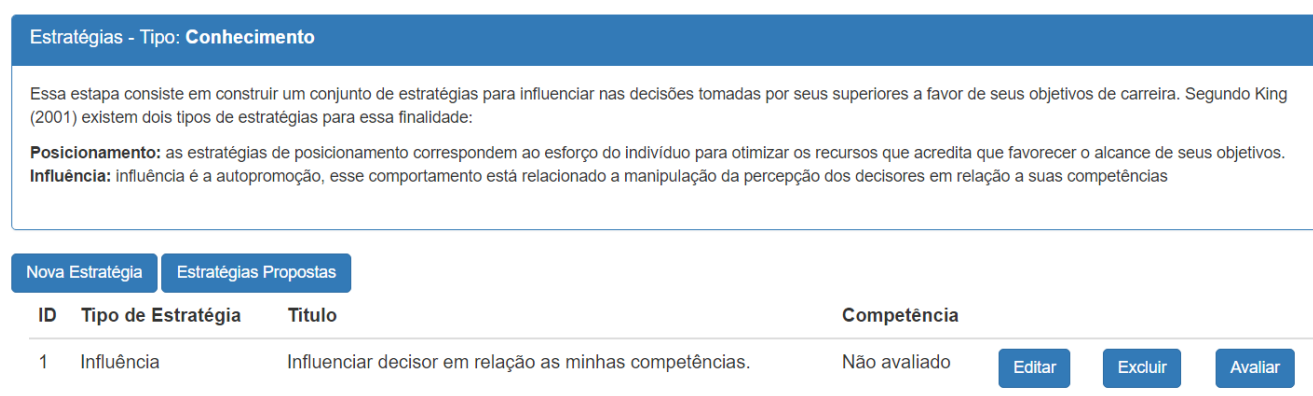

Figura 5: Exemplo de estratégia para atingimento de competências

Para cada estratégia personalizada, é necessário que o aluno faça uma autoavaliação, que permitirá identificar qual estratégia poderá ser mais eficaz. Esta autoavaliação é fundamental para a aprendizagem crítica e o uso de estratégias metacognitivas na gestão de carreiras. A autoavaliação leva em consideração: suas crenças de competência e sua opinião sobre regra de contingência. Esta etapa é bastante importante na medida em que dará pistas a seleção de estratégias futura. Para cada competência relacionada ao objetivo escolhido do aluno, o MaxMentor propõe algumas estratégias que têm como objetivo deixar que o aluno tome uma decisão em relação a suas competências e expectativas, podendo ser ela uma escolha correta ou não. $O$ MaxMentor propõe seis estratégias distintas para cada competência, de acordo com os requisitos profissionais identificados para a competência, atribuindo um grau de satisfação da competência para cada uma delas. Com isso o tutor tem todas as propriedades para conseguir fazer uma avaliação a partir das escolhas do aluno e trazer feedbacks positivos ou negativos em relação a esta escolha.

Após a implementação das estratégias, o tutor oferece um resumo de avaliação pessoal, mostrando como o aluno está no momento atual da tutoria de sua carreira. Nessa avaliação o sistema oferece totalizadores de competências, indicando a quantidade de situações-problema necessários para seu objetivo, bem como a quantas situações-problemas têm crenças positivas e quantas crenças negativas. Mostra também um resumo de estratégias escolhidas até o momento, indicando ao aluno como suas escolhas podem afetar na gestão de sua carreira. Quando o aluno faz uma escolha que vai contra seus objetivos, ou que pode ser mal vista pelo seu decisor, o MaxMentor orienta de forma que o aluno entenda que tais escolhas fazem com que as chances de alcançar seu objetivo diminuam. Estes indicadores são parte do processo de ensino de meta-cognição que é muito importante para gestão de carreiras, podendo ser acessados em qualquer momento da tutoria. Somente com a avaliação final indicativa que todas as situações-problema propostas foram resolvidas a contento e que o MaxMentor considera que a etapa atual do processo de tutoria de gestão de carreiras poderá ser finalizado.

\section{Protótipo do Sistema MaxMentor}

A Figura 6 mostra a arquitetura e a organização do protótipo do sistema MaxMentor. O protótipo do MaxMentor foi desenvolvido com uma arquitetura dividida no frontend (cliente) e no backend (servidor). O frontend foi desenvolvido em HTML5 e JavaScript. O backend está escrito completamente em SWI-Prolog, usando o pacote HTTP para implementar o servidor web que fornece as páginas web para o browser, e o módulo de 
VI Congresso Brasileiro de Informática na Educação (CBIE 2017)

Anais do XXVIII Simpósio Brasileiro de Informática na Educação (SBIE 2017)

interface de serviços web que atende as requisições destes serviços. O frontend acessa os serviços web do servidor por meio de requisições JQuery+AJAX. A comunicação de informações entre cliente e servidor é feita através do formato JSON, que tem suporte tanto em JavaScript quanto Prolog.



Figura 6: Arquitetura e Organização do Protótipo do MaxMentor

Além do servidor web e da interface de serviços web, a arquitetura do backend é baseada em quatro agentes de software. Cada agente foi criado como um módulo Prolog que se comunica por mensagens com os demais agentes e também com o módulo de interface de serviços web. As bases de conhecimentos de Perfis de Profissionais e de Carreiras foram definidas de acordo com a ontologia GPC. O agente Perfil do Profissional é responsável por obter informações do profissional (usuário) através da interface web e armazenar essas informações na base de conhecimento de Perfis de Profissionais. Este agente opera principalmente na primeira etapa do processo de tutoria. O agente Especialista em Carreira de TI é responsável pelo conhecimento da estrutura das carreiras de TI suportadas pelo MaxMentor. A Figura 7 mostra um pequeno trecho da base de Carreiras deste agente, definida de acordo com a ontologia GPC. Este agente fornece informações sobre as carreiras e funções para todos os outros agentes, além de gerenciar a base de conhecimento de carreiras.

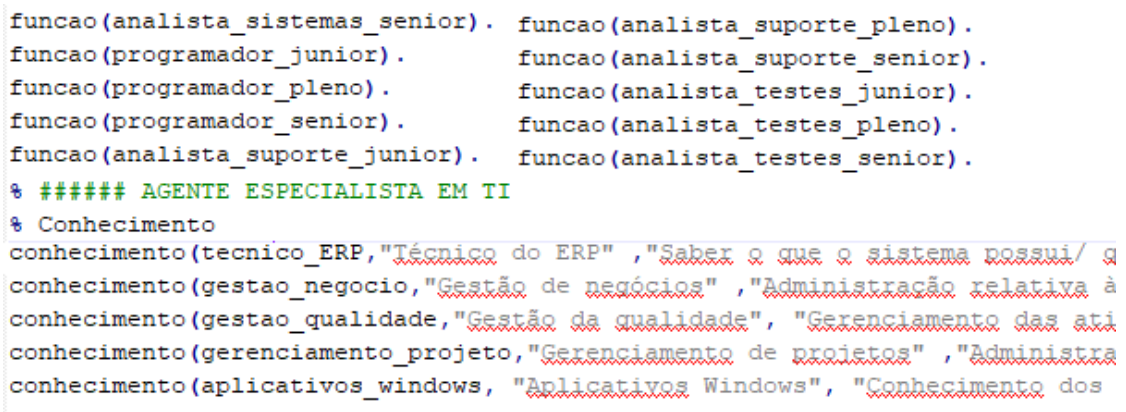

Figura 7: Exemplos de fatos da base de conhecimentos de carreiras

Os demais agentes são agentes mentores, ou seja, agentes responsáveis pela condução do processo de tutoria. O agente de Situações-Problema, é responsável por criar um plano de situações-problema específico para o aluno. A atuação pedagógica deste agente foi modelada por meio uma base de conhecimentos composta de fatos e regras lógicas divididas em um núcleo constituído de descrições genéricas de situaçõesproblema e de módulos específicos contendo regras que associam as situações-problema 
à carreiras e competências específicas. Os fatos e regras correspondentes a cada situação-problema foram obtidos a partir da análise do plano de carreiras, permitindo inferir logicamente as avaliações do mercado para cada competência. Com acesso as informações fornecidas pelos demais agentes, este agente é capaz de criar situaçõesproblemas a partir do perfil do profissional, dos seus objetivos de carreira e de informações relativas a estrutura e função de carreira. Por fim, o agente de Avaliação Crítica, é o agente responsável por implementar as inferências para avaliação de estratégias e de auxiliar o usuário em sua avaliação crítica das estratégias que pretende implementar para resolver as situações-problema.

\section{Experimentos e Resultados}

A avaliação do protótipo do MaxMentor foi realizada através de um cenário projetado para representar de forma realista uma situação onde a gestão da própria carreira se torna determinante. $\mathrm{O}$ cenário foi avaliado de forma experimental e empírica por um conjunto de cinco voluntários da área de TI que trabalham em uma empresa de médio porte da área de Informática. As idades dos voluntários estão entre vinte até quarenta anos e o tempo de experiência de cada um, varia de dez a vinte anos de trabalho.

É importante salientar a dificuldade de se fazer avaliações realistas de um ambiente como o MaxMentor. As situações que provocam mudanças de carreira tem um forte componente individual e não são algo corriqueiro na vida das pessoas. Para tentar contornar esse fator, o realismo buscado para o cenário de avaliação está fundamentado não apenas nas características do MaxMentor, mas também na experiência prévia dos voluntários: todos os voluntários selecionados passaram por uma experiência recente de mudança de função na área de TI, tendo uma boa noção dos desafios e problemas que esta situação pode causar. Além disso as características individuais de cada caso e a novidade do modelo King para os participantes, levaram a necessidade de uma forma restrita e técnica de pesquisa-ação (Tripp, 2005; Thiollent, 2009), para a condução dos experimentos. Segundo (Tripp, 2005) a pesquisa ação técnica é uma abordagem mais pontual de pesquisa-ação, na qual o pesquisador toma uma prática pré-existente e a aplica na sua própria esfera para realizar uma melhora. Ela é técnica porque o pesquisador age de forma inteiramente mecânica essencialmente seguindo o manual, ou, no caso da presente pesquisa, sendo efetivamente o manual do ambiente. A condução dos experimentos foi dividida em três partes: primeiro foi feita uma introdução ao MaxMentor. Logo após, os voluntários interagiram com o sistema com a ajuda do pesquisador, onde cada um trabalhou suas experiências recentes de troca de função.

Tabela 2: Utilidade do MaxMentor para aprendizagem sobre Gestão de Carreiras

\begin{tabular}{|c|c|c|c|c|c|}
\hline Questão & 1 & 2 & 3 & 4 & 5 \\
\hline $\begin{array}{l}\text { 1. A utilização do MaxMentor deixa claro as competências necessárias } \\
\text { para meus objetivos de carreira }\end{array}$ & & & & & $100 \%$ \\
\hline $\begin{array}{l}\text { 2. A utilização do MaxMentor melhora minha compreensão para criar } \\
\text { novas estratégias de carreiras }\end{array}$ & & & & $20 \%$ & $80 \%$ \\
\hline $\begin{array}{l}\text { 3. As situações-problemas do MaxMentor apresentam desafios realistas } \\
\text { para a gestão de carreiras }\end{array}$ & & & & $20 \%$ & $80 \%$ \\
\hline $\begin{array}{l}\text { 4. A avaliação pessoal proposta pelo MaxMentor deixa claro a situação } \\
\text { atual da minha carreira }\end{array}$ & & & & $40 \%$ & $60 \%$ \\
\hline
\end{tabular}

$\mathrm{Na}$ etapa final, cada voluntário respondeu um questionário de avaliação da usabilidade, baseado na metodologia TAM2 (Marangunić e Granić, 2015), levando em conta duas variáveis: a utilidade do sistema, em termos de aprendizagem sobre gestão de carreiras, e a facilidade de uso do sistema. Todas as questões foram formuladas como afirmações pontuadas pela escala Likert: 1 - discordo; 2 - discordo parcialmente; 3 sou indiferente; 4 - concordo parcialmente; 5 - concordo. A Tabela 2 apresenta os resultados da avaliação dos voluntários em relação a utilidade do MaxMentor para o aprendizado de técnicas de Gestão de Carreiras. Todos os resultados tiveram índice 
entre quatro e cinco na escala Likert, mostrando evidências muito boas da utilidade do sistema para auxiliar em suas vidas profissionais e na gestão da suas carreiras. $O$ resultado pode ser considerado muito bom, pois todos os índices estão acima de quatro, o que é considerado um alto índice de satisfação com a utilidade da tecnologia pela metodologia TAM2.

A Tabela 3 apresenta os resultados das questões sobre facilidade de uso da ferramenta. Os resultados desse conjunto de questões variam entre os índices três e cinco da escala Liker, o que também é considerado um alto índice de aceitação na metodologia TAM2. Apenas a questão 7, sobre a interface visual e o material explicativo, indica possibilidade de melhora. Assim os resultados dos experimentos mostram boas evidências em relação à facilidade de uso do MaxMentor.

Tabela 3: Facilidade de uso da ferramenta.

\begin{tabular}{|c|c|c|c|c|c|}
\hline Questão & 1 & 2 & 3 & 4 & 5 \\
\hline 5. As situações-problemas são de fácil entendimento & & & & $20 \%$ & $80 \%$ \\
\hline 6. Aprender a utilizar o MaxMentor é fácil & & & $20 \%$ & $20 \%$ & $60 \%$ \\
\hline 7. Os menus, opções, ícones e o texto são de fácil compreensão & & & & $60 \%$ & $40 \%$ \\
\hline 8. A organização dos conteúdos permite navegação dinâmica e fácil & & & & $75 \%$ & $25 \%$ \\
\hline
\end{tabular}

A avaliação da questão de pesquisa do MaxMentor que envolve a possibilidade deste IMS auxiliar na aprendizagem de estratégias meta-cognitivas é particularmente difícil em termos empíricos: perguntas aos participantes dos experimentos resultariam nas crenças subjetivas a respeito do assunto não necessariamente relacionadas com a possibilidade deste sujeito aplicar efetivamente estratégias meta-cognitivas. O MaxMentor fornece alguma evidência deste processo porque para avançar até o fim das situações-problemas, o usuário deve confrontar suas crenças de competência com indicadores concretos de competência. Mas a avaliação empírica sistemática dependeria de um processo estendido de observação dos voluntários que buscaria evidências concretas do uso de estratégias meta-cognitivas. Devido a impossibilidade de recursos e de tempo para tal fim optou-se pela consulta a uma especialista de coaching de carreiras. Esta especialista é formada em psicologia, com MBA em Gestão de Pessoas pela FGV (Fundação Getúlio Vargas) e curso de Coaching Executivo e Empresarial pela ABRACEM (Associação Brasileira de Coaching Executivo e Empresarial).

A especialista teve acesso pleno ao sistema MaxMentor, recebendo o mesmo tipo de orientação de uso do sistema que os demais voluntários. A especialista também teve acesso aos resultados de avaliação dos voluntários. Ao final do uso experimental do sistema foi apresentado um questionário para avaliar a qualidade do material de tutoria e o impacto que o uso do MaxMentor teria no pensamento crítico sobre conhecimentos e competências. A três questões iniciais sobre a qualidade do conteúdo avaliavam se: 1) os métodos usados para gestão de carreira (Modelo King, Competências CHA e SituaçõesProblema) ajudam na carreira individual, 2) as funções abordadas no sistema cobrem as competências necessárias para cada uma delas e 3) os materiais oferecidos pelo sistema estão próximos da realidade atual. Nessas questões a especialista em coaching concordou plenamente com a utilidade dos métodos no MaxMentor, tal como afirmado na questão 1, e concordou parcialmente com as afirmações das questões 2 e 3. A avaliação de impacto se dividiu em questões que verificavam se: 4) o uso do MaxMentor pode ajudar um individuo a gerir sua carreira e identificar conhecimentos a serem atingidos, 5) o uso do MaxMentor pode ajudar um individuo a gerir sua carreira e identificar competências a serem atingidas e 6) o MaxMentor pode ajudar um individuo a pensar criticamente sobre sua carreira, levando-o a analisar criticamente seus conhecimentos e competências em relação aos seus objetivos de carreira. Em relação a essas questões houve uma concordância plena da especialista com todas as afirmações das questões de 4 a 6 . Apresentando bons resultados, essa avaliação é muito importante para o presente trabalho trazendo evidências de que o MaxMentor pode ajudar no pensamento crítico sobre como gerir a própria carreira. 
VI Congresso Brasileiro de Informática na Educação (CBIE 2017)

Anais do XXVIII Simpósio Brasileiro de Informática na Educação (SBIE 2017)

\section{Conclusões}

O sistema de tutoria MaxMentor combina um conjunto de técnicas avançadas, incluindo ontologias, sistemas de inferência lógica e tecnologia de agentes, para prover um ambiente inteligente de ensino de estratégias de gestão pessoal de carreiras. O objetivo pedagógico que diferencia o MaxMentor de outros sistemas similares de "mentoria", é sua utilização de situações-problema não apenas para orientar o processo de aprendizagem dessas estratégias, mas também para permitir ao profissional (aluno) desenvolver suas capacidades de pensamento crítico a respeito da sua forma de gestão da carreira, ou seja, desenvolver estratégias meta-cognitivas para lidar com essa gestão. Os experimentos empíricos realizados com o MaxMentor trazem evidências de um impacto positivo do MaxMentor na gestão pessoal da carreira. A avaliação deste sistema por um especialista em coaching de carreiras também traz evidências positivas de que o MaxMentor pode ajudar na meta-cognição. O caminho das pesquisas futuras envolve, além de experimentos empíricos de mais longo prazo para observar se estas evidências positivas realmente persistem, a evolução do sistema para possibilitar a interação do sistema usando linguagem natural, o que permitiria uma interface muito mais livre, rica e próxima do mercado em opções de estratégias para alavancar competências.

\section{Referências}

Carbone, P. P.; Brandão, H.P.; Leite, J.B.D.; Vilhena, R.M.P. (2009) Gestão por Competências e Gestão do Conhecimento. ed. 3. Rio de Janeiro: FGV.

Crites (1976) A comprehensive model of career development in early adulthood, Journal of Vocational Behavior, 9, 105-188.

Dimitrova1,V.; Poulovassilis, A.; Labeke1, N.; Treasure-Jones, T.; Zukas, M.; Brna, P. (2016). Intelligent Mentoring Systems for Making Meaning from Work Experience (IMS-2016) - International Conference of Intelligent Tutoring Systems (ITS-2016).

Ferreira, Aurélio B. de Hollanda. Novo Dicionário da Língua Portuguesa. 8. ed. Rio de Janeiro: Nova Fronteira, 2010.

King, Z. (2001) Career self-management: a framework for guidance of employment adults. British Journal of Guidance \& Counselling.

King, Z. (2004) Career self-management: its nature, causes and consequences. Journal of Vocational Behavior.

Marangunić, N., Granić, A. (2015) Technology Acceptance Model: A Literature Review from 1986 to 2013. v. 14, n. 1, p. 81-95.

Oliveira, S., Gluz, J.C. (2010) Agent-Based Cognitive Model for Human Resources Competence Management. Advances in Experimental Medicine and Biology.

Oluwabunmi, A., Fred, P., Julita, V. (2016) Dynamics of Trust in Group Peer Mentorship. Proceedings of 1st International Workshop on Intelligent Mentoring Systems (IMS-2016) - International Conference of Intelligent Tutoring Systems (ITS-2016).

Palacios, R. C., Lumbreras, C. C. (2006) Mentoring \& Coaching. IT Perspective. Journal of Technology Management \& Innovation.

Pinto, J.C.N. (2010) Gestão Pessoal da Carreira Estudo de um Modelo de Intervenção Psicológica com Bolseiros de Investigação, Dissertação. Univ. do Minho, Portugal.

Ragins, B. R.; Kram, E. D. (2007). The handbook of mentoring at work: theory, research and practice. Thousand Oaks, CA: Sage.

Rosa, J. H.; Barbosa, J. L. V.; Kich, M. R.; Brito, L. K. A. (2015) Multi-Temporal Contextaware System for Competences Management. International Journal of Artificial Intelligence in Education.

Thiollent, M. Metodologia da pesquisa-ação. São Paulo: Cortez, 2009.

Trip, D. Pesquisa-ação: uma introdução metodológica. Educação e Pesquisa, São Paulo, v. 31, n. 3, p. 443-466, set./dez. 2005.

Weerasinghe, A.; Dimitrova, V.; Lau, L.; Mitrovic, A. (Chairs) (2016). Proceedings of 1st International Workshop on Intelligent Mentoring Systems (IMS-2016) - International Conference of Intelligent Tutoring Systems (ITS-2016). 